\title{
Notes on the blood-feeding behavior of Aedes albopictus (Diptera: Culicidae) in Cameroon
}

\author{
Basile Kamgang ${ }^{1,2,3}$, Elysée Nchoutpouen ${ }^{1}$, Frédéric Simard ${ }^{1}$ and Christophe Paupy ${ }^{1,4^{*}}$
}

\begin{abstract}
Background: The invasive mosquito Aedes albopictus is often considered a poor vector of human pathogens, owing to its catholic feeding behavior. However, it was recently incriminated as a major vector in several Chikungunya epidemics, outside of its native range. Here we assessed two key elements of feeding behavior by Ae. albopictus females in Yaoundé, Cameroon, Central Africa. Host preference was explored and the human-biting activity of females was monitored over $24 \mathrm{~h}$ to determine periods of maximum bite exposure.

Findings: Analysis of ingested blood in outdoor-resting females showed that Ae. albopictus preferentially fed on humans rather than on available domestic animals (95\% of the blood meals contained human blood). Our results further showed that Ae. albopictus is a day-biting species in Yaoundé, with a main peak of activity in the late afternoon.

Conclusion: This is the first report on the feeding behavior of Ae. albopictus in Central Africa. The species is highly aggressive to humans and might therefore be involved in human-human virus transmission in this setting.
\end{abstract}

Keywords: Aedes albopictus, Feeding behavior, Host preference, Nychthemeral activity, Cameroon

\section{Background}

Aedes albopictus (Skuse, 1894) is an invasive mosquito species that originated in Asian forests [1] and expanded to temperate and tropical regions of America, Africa and Europe, mainly during the last three decades [2]. Aedes albopictus is widely considered as a secondary vector of human arboviruses such as dengue virus (DENV), because it is thought to preferentially feed on animals rather than humans, contrary to the highly anthropophilic Aedes aegypti (L., 1762) [3]. Yet, Ae. albopictus has been incriminated as a primary vector in recent chikungunya virus (CHIKV) epidemics in the Indian Ocean, Central Africa and Europe [4-6], suggesting sustained man-vector contact in these settings. In Cameroon (Central Africa), Ae. albopictus was first recorded in the early 2000s [7] and rapidly spread throughout the south of the country [8]. In Cameroonian urban centers such as Yaoundé, the species now pullulates [9] and is gradually replacing autochthonous Ae. aegypti populations. Concomitant with the spread of Ae. albopictus in Central Africa, an increase in DENV and

\footnotetext{
* Correspondence: christophe.paupy@ird.fr

'Institut de Recherche pour le Développement (IRD), UMR MIVEGEC (UM1CNRS 5290-IRD 224), team BEES, P.O. Box 64501, Montpellier 34394, France Full list of author information is available at the end of the article
}

CHIKV outbreaks was reported in a number of countries, including Cameroon [10], Gabon [5,11] and the Republic of Congo [12]. Indeed, Cameroonian Ae. albopictus populations were shown to be orally susceptible to DEN-2 virus and CHIKV infection [5], and the species was recognized as the main vector of both viruses in 2007 in Libreville, Gabon [5,11].

Although Ae. albopictus preferentially bites mammals [3], females can also feed on most groups of vertebrates, both cold- and warm-blooded, including reptiles, birds and amphibians [13]. Such feeding plasticity, which has been found to vary according to the geographic origin of mosquito populations [14], maximizes the fitness (fecundity and survival) of Ae. albopictus and enhances the risk that it may propagate zoonotic pathogens from wildlife or domestic animals to humans [15]. In addition to host preferences, the intensity of human-vector contact and, therefore, the risk of pathogen transmission, is modulated by the biting period(s) along the nychthemere, which impacts on host availability. Although geographic variations have been recorded in the biting rhythm of Ae. Albopictus females, the species usually bites outdoors and during daytime, with two main peaks of activity in the morning and early evening $[3,4,14]$. To our knowledge, 
there are no published data on the feeding behavior of Ae. albopictus in Central Africa. In order to fill this gap, we conducted a study of Ae. albopictus host preferences and biting activity in Yaoundé, Cameroon.

\section{Methods}

Mosquitoes were collected from two sites in the periphery of Yaoundé, the capital city of Cameroon: "Club France" (N3 $\left.50^{\prime} 28^{\prime \prime}, \mathrm{E} 11^{\circ} 30^{\prime} 2^{\prime \prime}\right)$ in the Efoulan district and "Club hippique" (N3 ${ }^{\circ} 4^{\prime} 05^{\prime \prime}, \mathrm{E} 11^{\circ} 29^{\prime} 53^{\prime \prime}$ ) in the Ntougou district. "Club France", a leisure center (tennis, swimming pool, banquets, etc.;), is surrounded by dense vegetation which provides potential resting and oviposition sites for Aedes mosquitoes. "Club hippique", an equestrian center, is also surrounded by dense vegetation. The horses $(\mathrm{N}>25)$ provide potential blood sources for mosquitoes. Additionally, both sites are surrounded by human dwellings with domestic animals such as dogs, pigs and chickens.

Field collections were conducted from April to early July 2009, during the rainy season when mosquito densities are highest. To determine the feeding preferences of Ae. albopictus, wild blood-fed females were caught in both study sites. The low vegetation that constitutes resting sites was stirred up to disturb mosquitoes, and flying females were caught by sweep-netting. Each capture was done by a single collector and lasted $30 \mathrm{~min}$. Collected mosquitoes were individually transferred to 10 - $\mathrm{mL}$ glass vials using a mouth aspirator; they were identified morphologically by reference to standard keys [16] and stored at $-20^{\circ} \mathrm{C}$ until blood meal analysis. The abdomen of freshly-fed Ae. albopictus and Ae. aegypti females was dissected out and used as a template for blood-meal analysis by ELISA [17], using anti-human, -horse, -bird, -reptile, -swine, -sheep and -dog antibodies (Sigma Aldrich, St. Louis, MO). To explore the biting activity of Ae. albopictus, blood-seeking females were trapped outdoors over entire 24-hour periods using a double-net device baited with a human volunteer, as described in Delatte et al. [14]. The experiment was repeated eight times in "Club France" only, between April and July 2009.

\section{Results and discussion}

In "Club France", a total of 537 resting Aedes females were harvested during 46 capture sessions, of which 479 were Ae. albopictus (including 139 freshly fed) and 58 Ae. aegypti (including 6 freshly fed). Twenty-one capture sessions in "Club hippique" recovered 77 Ae. albopictus females (including 31 freshly fed) and no Ae. aegypti. Males of either species were observed but not collected, as well as other mosquito genera such as Culex. ELISA analyses indicated that $A$ e. albopictus specimens had primarily fed on human blood, as about $95 \%$ of dissected stomachs contained human blood in the two sites (Table 1). Mixed meals of human and reptile or pig blood were also
Table 1 Ingested-blood analysis in Ae.albopictus females from sites in Yaoundé, Cameroon

\begin{tabular}{lll}
\hline Host & \multicolumn{2}{l}{ Number of specimens analyzed (\% positive) } \\
\hline Human & $130(93.5)$ & Club hippique \\
\hline Reptile & $1(0.7)$ & $26(83.9)$ \\
\hline Horse & 0 & 0 \\
\hline Chicken & 0 & 0 \\
\hline Dog & 0 & 0 \\
\hline Sheep & 0 & 0 \\
\hline Swine & 0 & 0 \\
\hline Human-Reptile & $2(1.5)$ & 0 \\
\hline Human-Swine & 0 & 0 \\
\hline No-call & $6(4.3)$ & $3(9.7)$ \\
\hline Total & $139(100)$ & $2(6.4)$ \\
\hline
\end{tabular}

${ }^{\mathrm{a}}$ No reaction with antibodies to any of the tested hosts.

observed. All 6 Ae. aegypti females collected from "Club France" had fed on human blood. These results conflict with the assumption that Ae. albopictus is mainly zoophilic $[2,3,13]$, and are consistent with observations made in Thailand [18], North Carolina (United States) [19], Italy [20] and La Réunion [14]. Our results are all the more convincing as animals were available in both study sites, showing that Ae. albopictus prefers to feed on humans, at least in Yaoundé. This propensity of Ae. albopictus females to feed on humans in urban areas of Cameroon is of concern, as it suggests a risk of human-human pathogen transmission. Moreover, the observation of blood meals from pigs and reptiles, and especially mixed animalhuman meals, although few in number, confirms that this species could act as a bridge vector for zoonotic pathogens. Furthermore, in about $5 \%$ of cases the host species could not be identified, suggesting either advanced digestion of the blood or the existence of unidentified animal hosts such as rodents.

Mosquito collection using a double-net device demonstrated that Ae. albopictus females feed during daytime from 5:00 a.m. to 7:00 p.m. with a main peak from 3:00 p. m. to 7:00 p.m (Figure 1). These results are consistent with the known biology of Ae. albopictus outside Africa $[3,14,21]$. Although Ae. albopictus is sometimes observed indoors, it is generally considered as exophilic and exophagic [4]. In their recent work in La Réunion Island (France, Indian Ocean), using a similar double-net collection device placed indoors and outdoors, Delatte et al. [14] estimated that $89 \%$ of mosquitoes were exophagic. Our results, based solely on outdoor collections, therefore, likely reflect the major biting pattern of Ae. albopictus females in this area. However, further studies are required to fully explore the feeding behavior of invasive populations of Ae. albopictus and to assess their role in the emergence and spread of 


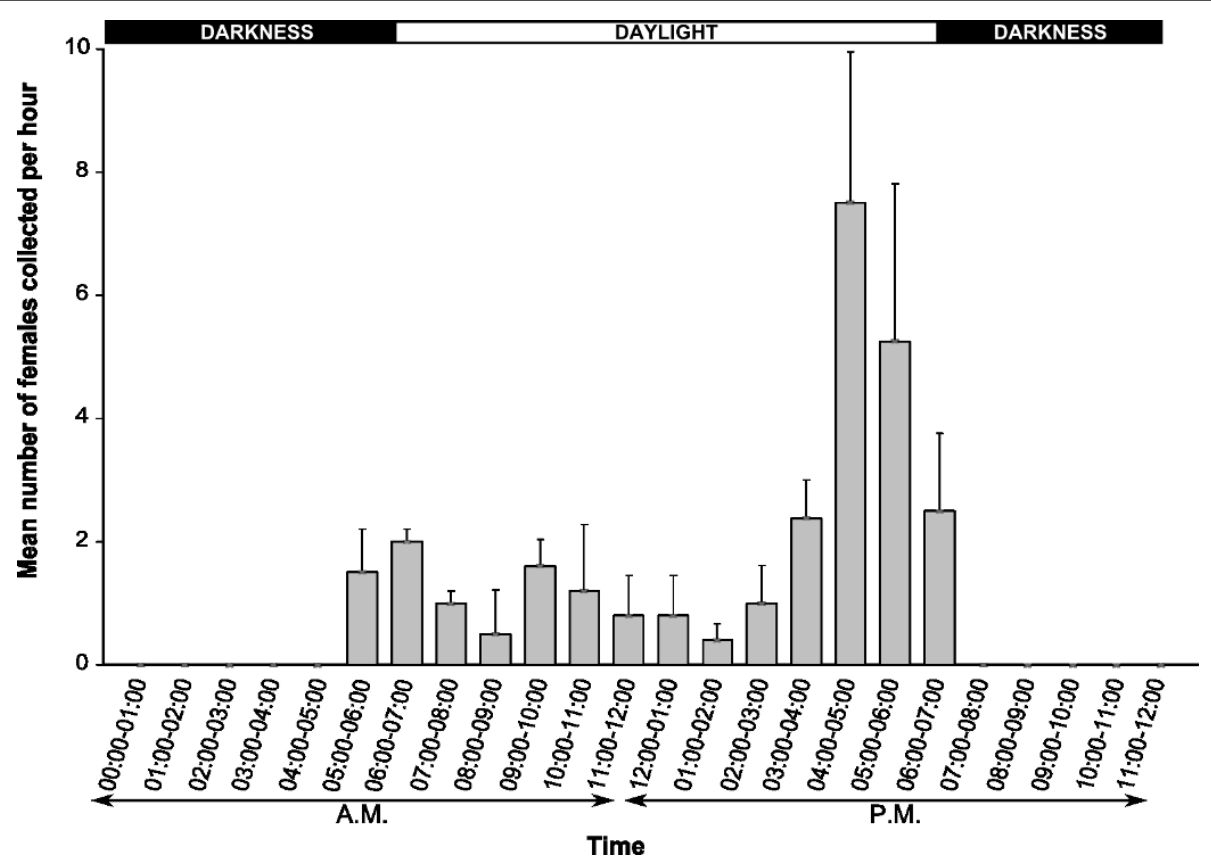

Figure 1 Daily dynamics of host-seeking activity in Ae. albopictus females from Yaoundé, Cameroon (April-July 2009). Vertical bars represent standard deviation.

arbovirus epidemics in Africa and elsewhere, including areas where Ae. aegypti is the dominant species.

\section{Conclusion}

This study, which provides the first data on the feeding behavior of Ae. albopictus in Central Africa, demonstrates that this species readily bites humans in urban settings and might therefore play a major role in humanhuman transmission of emerging arboviruses such as DENV and CHIKV. Detailed knowledge of the feeding behavior of this vector species, such as the rhythm of females' biting activity, is needed to develop and implement efficient personal protection tools against Ae. albopictus, especially in newly colonized areas.

\section{Acknowledgements}

We thank Rose Nyambam for technical assistance with ELISA analysis. This research was supported by the French government through "Agence Nationale pour la Recherche", that funded the Epidengue project (ANR 05 SEST 010-01). Basile Kamgang was supported by an IRD/DSF PhD fellowship.

\section{Author details}

${ }^{1}$ Institut de Recherche pour le Développement (IRD), UMR MIVEGEC (UM1CNRS 5290-IRD 224), team BEES, P.O. Box 64501, Montpellier 34394, France. ${ }^{2}$ Laboratoire de Recherche sur le Paludisme, Organisation de Coordination pour la lutte contre les Endémies en Afrique Centrale (OCEAC), P.O. Box 288, Yaoundé, Cameroon. ${ }^{3}$ Institut Pasteur de Bangui, P.O. Box 923, Bangui, Republic of Central Africa. ${ }^{4}$ Centre International de Recherches Médicales de Franceville (CIRMF), P.O. Box 769, Franceville, Gabon.

\section{Authors' contributions}

BK and CP designed the study and monitored its implementation. BK and EN conducted the field work. BK analyzed the data. BK and CP wrote the manuscript which was critically revised by FS. All authors read and approved the final manuscript

\section{Competing interests}

The authors declare that they have no competing interests.

Received: 12 January 2012 Accepted: 21 March 2012

Published: 21 March 2012

\section{References}

1. Smith CEG: The history of dengue in tropical Asia and its probable relationship to the mosquitoes Aedes aegypt. J Trop Med Hyg 1956, 59:3-11.

2. Gratz NG: Critical review of the vector status of Aedes albopictu. Med Vet Entomol 2004, 18:215-227.

3. Hawley AH: The biology of Aedes albopictu. J Am Mosq Control Assoc 1988, 4:2e39.

4. Paupy C, Delatte H, Bagny L, Corbel V, Fontenille D: Aedes albopictu, an arbovirus vector: from the darkness to the light. Microbes Infect 2009, 11:1177-85.

5. Paupy C, Ollomo B, Kamgang B, Moutailler S, Rousset D, Demanou M, Hervé JP, Leroy E, Simard F: Role of Ae. albopictus versus Ae. aegypti in dengue and chikungunya emergences in Central Africa: laboratory and field evidence. Vector Borne and Zoonotic Dis 2010, 10(3):259-66.

6. Pagès F, Peyrefitte CN, Mve MT, Jarjaval F, Brisse S, Iteman I, Gravier P, Tolou H, Nkoghe D, Grandadam M: Aedes albopictu mosquito: the main vector of the 2007 Chikungunya outbreak in Gabon. PLOS One 2009, 4(3): e4691.

7. Fontenille D, Toto JC: Aedes (Stegomyia) albopictu (Skuse), a potential new dengue vector in Southern Cameroon. Emerg Infect Dis 2001, 7:1066-1067.

8. Simard F, Nchoutpouen E, Toto JC, Fontenille D: Geographic distribution and breeding site preference of Aedes albopictu and Aedes aegypt (Diptera: Culicidae) in Cameroon, Central Africa. J Med Entomol 2005, 42:726-731.

9. Kamgang B, Happi JY, Boisier P, Njiokou F, Hervé JP, Simard F, Paupy C: Geographic and ecological distribution of the dengue and chikungunya 
virus vectors Aedes aegypt and Aedes albopictu in three major Cameroonian towns. Med Vet Entomol 2010, 24(2):132-41.

10. Peyrefitte CN, Rousset D, Pastorino BA, Pouillot R, Bessaud M, Tock F, Mansaray H, Merle OL, Pascual AM, Paupy C, Vessiere A, Imbert P, Tchendjou P, Durand JP, Tolou HJ, Grandadam M: Chikungunya virus, Cameroon, 2006. Emerg Inf Dis 2007, 13:768-771.

11. Leroy EM, Nkoghe D, Ollomo B, Nze-Nkogue C, Becquart P, Grard G, Pourrut X, Charrel R, Moureau G, Ndjoyi-Mbiguino A, De-Lamballerie X: Concurrent chikungunya and dengue virus infections during simultaneous outbreaks, Gabon. Emerg Inf Dis 2009, 15:591-593.

12. Kelvin AA: Outbreak of Chikungunya in the Republic of Congo and the global picture. J Infect Dev Ctries 2011, 5(6):441-444.

13. Scholte EJ, Schaffner F: Waiting the tiger: establishment and spread of the Aedes albopictus mosquito in Europe. In Emerging Pests and VectorBorne Diseases in Europe. Edited by: Takken W, Knols BGJ. Wageningen: Wageningen Academic Publishers; 2007:241-260.

14. Delatte H, Desvars A, Bouétard A, Bord S, Gimonneau G, Vourc'h G, Fontenille D: Blood-feeding behavior of Aedes albopictu, a vector of Chikungunya on La Réunion. Vector Borne Zoonotic Dis 2010, 10(3):249-58.

15. Gubler DJ: Aedes albopictu in Africa. Lancet Infect Dis 2003, 3:751-752.

16. Jupp PG: Mosquitoes of Southern Africa: Culicinae and Toxorhynchitinae Hartebeespoort, South Africa: Ekogilde Publishers; 1996, 156.

17. Beier JC, Perkins PV, Wirtz RA, Koros J, Diggs D, Gargam-II TP, Koech DK: Bloodmeal identification by direct enzyme-linked immunosorbent assay (ELISA) tested on Anopheles (Diptera: Culicidae) in Kenya. J Med Entomol 1988, 25:9-16.

18. Ponlawat A, Harrington LC: Blood feeding patterns of Aedes aegypt and Aedes albopictu in Thailand. J Med Entomol 2005, 42(5):844-9.

19. Richards SL, Ponnusamy LT, Unnasch TR, Hassan HK, Apperson CS: HostFeeding patterns of Aedes albopictu (Diptera: Culicidae) in relation to availability of human and domestic animals in suburban landscapes of central North Carolina. J Med Entomol 2006, 43(3):543-551.

20. Valerio L, Marini F, Bongiorno G, Facchinelli L, Pombi M, Caputo B, Maroli M, Della Torre A: Host-feeding patterns of Aedes albopictu (Diptera: Culicidae) in urban and rural contexts within Rome province, Italy. Vector Borne Zoonotic Dis 2010, 10(3):291-4.

21. Almeida AP, Baptista SS, Sousa CA, Novo MT, Ramos HC, Panella NA, Godsey M, Simões MJ, Anselmo ML, Komar N, Mitchell CJ, Ribeiro H: Bioecology and vectorial capacity of Aedes albopictus (Diptera: Culicidae) in Macao, China, in relation to dengue virus transmission. J Med Entomol 2005, 42(3):419-28.

doi:10.1186/1756-3305-5-57

Cite this article as: Kamgang et al:: Notes on the blood-feeding behavior of Aedes albopictus (Diptera: Culicidae) in Cameroon. Parasites \& Vectors 2012 5:57.

\section{Submit your next manuscript to BioMed Central and take full advantage of:}

- Convenient online submission

- Thorough peer review

- No space constraints or color figure charges

- Immediate publication on acceptance

- Inclusion in PubMed, CAS, Scopus and Google Scholar

- Research which is freely available for redistribution

Submit your manuscript at www.biomedcentral.com/submit
Biomed Central 